\title{
REMARKS ON ZIL'BER'S RESULTS : ON THE SIMPLICITY OF THE REPRESENTATION OF GROUPS AND RINGS
}

\author{
By
}

\author{
Hirotaka KIKYo
}

\section{§ 0. Introduction.}

B. I. Zil'ber made tremendous amount of important works about groups of finite Morley rank. One of the most important works of his is the indecomposability theorem for groups of finte Morley rank, with which he proved that the simplicity of a group of finite Morley rank is preserved by elementary equivalence, and simple groups of finite Morley rank are almost strongly minimal.

We will show with his methods that the simplicity (or irreducibility) of a representation of a connected group or ring (not necessarily associative) over a group of finite Morley rank is preserved by elementary equivalence. As the simplicity of a group or a ring can be considered as the simplicity of its representation on itself, this extends the result of Zil'ber above and also we get the theorem for a ring of finite Morley rank. Berlin and Lascar extended the indecomposability theorem to the superstable case and one of their applications was the preservation theorem for the simplicity of a superstable group ([3]). We can also extend their works to the cases as here with some modification.

We also prove that a simple ring of finite Morley rank is almost strongly minimal. For a simple representation of a group or a ring, we can prove that it is strongly minimal under some stronger assumption and in a different language from above. Our results yield many algebraic examples of the almost strongly minimal structure, hence of the $|L|^{+}$-categorical structure where $L$ is the language of the structure considering. As for $\omega_{1}$-categorical ring, Zil'ber made complete classification for associative ring of characteristic zero ([8]), and Rose proved that finite dimensional central algebra over an algebraically closed field is $\omega_{1}$-categorical $([6])$. We can easily find an almost strongly minimal ring which is not associative or central, namely a finite dimensional simple Lie algebra for example.

Received December 25, 1986. Revised May 6, 1987. 


\section{$\S 1$. Preliminaries.}

We assume the readers to have some knowledge of stability theory in model theory and of stable groups. Our notations are fairly standard. Now we explain some notions and facts we need.

A pair of sets $(\Omega, G)$ is called a group with an operator domain $\Omega$ if $G$ is a group and each element of $\Omega$ acts on $G$ as a group endomorphism. In this case, we also call $G$ an $\Omega$-group or $(\Omega, G)$ a representation of $\Omega$ over $G$ and $G$ the representation space.

Let us now explain the rings we consider later. A structure $(R,+, \cdot)$ is called a ring if $(R,+)$ is a group and for each elements $a, b, c$ of $R, a(b+c)=$ $a b+a c$ and $(b+c) a=b a+c a$ holds. We will not assume the multiplication to be associative. Even we do not have to assume the operation "+" to be commutative neither. But the identity element of $(R,+)$ will be denoted by 0 . We can define ideals (left, right, or both-sided) and the simplicity of a ring in a same way as usual ones.

In this paper, we assume that $\Omega, G$ and the action of $\Omega$ on $G$ are definable (interpreted) in some structure $M$. If we were talking about definable subsets or ranks of $\Omega$ or $G$, then they are relative to $\operatorname{Th}(M)$. So, in every results below, $\Omega$ or $G$ can have other structure which is not appearlant. Also, definable will mean that definable with some parameters.

A subgroup of an $\Omega$-group $G$ is called $\Omega$-admissible or an $\Omega$-subgroup if it is closed under the action of $\Omega$. An $\Omega$-group $G$ is called simple or $\Omega$-simple if it contains no non-trivial $\Omega$-subgroups, and called definably simple if it contains no non-trivial definable $\Omega$-subgroups. Note that a group $G$ is simple if and only if $G$ is simple as a $G$-group where the action is given by taking conjugate. Also, a ring $R$ is simple if it is simple as an $R$-group or an $R \cup R^{\prime}$-group where $R^{\prime}$ is a copy of $R$ with some adequate action which will be clear depending on the definition of an ideal dealing with, left, right, or both-sided.

We give briefly important definitions and facts which are due to Zil'ber.

A definable subset $S$ of a group $G$ is called indecomposable if for any definable subgroup $H$ of $G$ if $S$ is covered by a finite number of cosets of $H$ then $S$ is covered by one of them. In this definition, if $S$ is a subgroup then $S$ is called connected. Note that the connectedness of a definable subset $S$ of $G$ is preserved by elementary equivalence with respect to the parameters used to define $S$ and $G$.

Proposition 1.1. Let $G_{1}, G_{2}$ be groups and $f: G_{1} \rightarrow G_{2}$ a definable homomorphism. If a definable subset $S$ of $G_{1}$ is indecomposable then $f(S)$ is indecom- 
posable.

This is a corollary to V.1.4 in [3] which was given as an exercise. Direct proof is also an easy exercise, but this one is an important fact for us. The following theorem of Zil'ber is a crucial lemma. A proof for it is available in [3] or [5].

Zil'ber's Indecomposability Theorem. Let $F$ be a family of indecomposable subsets of a group $G$ of finite Morley rank. Suppose each set in $F$ contains the identity element of $G$. Then $\langle U F\rangle$ is connected and equal to $X \cdot X^{-1}$ where $X=S_{1} \cdots S_{n}$ with $S_{i}$ 's from $F$.

\section{§2. Main Theorems.}

We state and prove our results in this section.

LEMmA 2.1. Let $G$ be an $\Omega$-group and $R M(G)<\omega$. If $S_{0}$ is an indecomposable subset of $G$ containing the identity element of $G$ then the $\Omega$-subgroup of $G$ generated by $S_{0}$ is a definable connected subgroup of $G$ which is algebraic over $S_{0}$ and some finite number of constants. In particular, if $G$ is $\Omega$-simple then $G$ is connected.

Proof. Define families of sets $F_{i}(i<\omega)$ by induction as follows :

$$
F_{0}=\left\{S_{0}\right\} \quad \text { and } F_{i+1}=\left\{S^{\theta}: \theta \in \Omega, S \in F_{i}\right\} .
$$

Put $F=\bigcup_{i<\omega} F_{i}$. Then every element of $F$ is indecomposable by Proposition 1.1 and contains the identity of $G$. Thus by the indecomposability theorem, $\langle\cup F\rangle=$ $X \cdot X^{-1}$ with $X=S_{1} \cdots S_{n}$ for some $S_{1}, \cdots, S_{n} \in F$. Hence, $\langle\cup F\rangle$ is the $\Omega$-subgroup generated by $S_{0}$, definable, connected and algebraic over $S_{0}$ and parameters used to define $S_{0}, S_{1}, \cdots, S_{n}$.

LemmA 2.2. Let $G$ be an $\Omega$-group. Suppose that $a^{\Omega}$ is indecomposable for each element $a$ of $G$, and $\left\{x \in G: \forall y, z \in \Omega\left(x^{y}=x^{z}\right)\right\}$ is $\Omega$-admissible and not equal to $G$. If $R M(G)<\omega$ and $G$ is definably $\Omega$-simple then $G$ is $\Omega$-simple.

Proof. Let $A$ be an $\Omega$-subgroup of $G$. We show that $A=\{e\}$ or $A=G$. Choose $\sigma \in \Omega$ and fix it. By the indecomposability theorem, the set $S_{0}=$ $\left\langle a^{\Omega}\left(a^{\sigma}\right)^{-1}\right\rangle_{a \in A}$ is connected, definable and contained in $A$. Consider the $\Omega$-subgroup generated by $S_{0}$. Note that it is a subgroup of $A$ and definable by Lemma 2.1. If it is not $\{e\}$ then it is equal to $G$, and hence $A=G$. If it equals to $\{e\}$ then $a^{\Omega}=a^{\sigma}$ for each $a \in A$. Hence, $A \subset\left\{x \in G: \forall y, z \in \Omega\left(x^{y}=x^{z}\right)\right\}=\{e\}$. 
We remark that the set $\left\{x \in G: \forall y, z \in \Omega\left(x^{y}=x^{z}\right)\right\}$ is the center of $G$ if $\Omega=G$ and $x^{y}=y x y^{-1}$, and also it becomes the (left, right) annihilator if a ring is considered.

Now we consider an $\Omega$-group where $\Omega$ has a structure of group.

Definition. Let $G$ be an $\Omega$-group where $(\Omega, *)$ is a group. $(\Omega, G)$ will be called a representation of type A if $\Omega$ acts on $G$ as a subgroup of the automorphism group of $G$, and a representation of type $B$ if for each $x, y \in \Omega$ and $a \in G, a^{x * y}=\left(a^{x}\right)\left(a^{y}\right)$ holds.

For example, a representation of a group is of type $A$ and a representation of a ring is of type $B$. There are two trivial actions of a group $\Omega$ on a group $G$, one of type $A$ and one of type $B$. Namely, if each element of $\Omega$ acts on $G$ as the identity map then it is of type $A$, and if each element of $\Omega$ sends every element of $G$ to its identity element then it is of type $B$.

THEOREM A. For a non-trivial representation of type $A$ or $B$ of a connected group, if the representation space is of finite Morley rank then if it is definably simple then it is simple. In particular, the simplicity is preserved by elementary equivalence.

ProOF. Let $(\Omega, G)$ be a representation of type $A$ or $B$. We will check that the assumption of Lemma 2.2 holds. We will denote the identity elements of both $\Omega$ and $G$ by the same letter $e$.

In the case that $(\Omega, G)$ is of type $A$, we can show that $a^{\Omega}$ is indecomposable for each $a \in G$ in the same way as the case that $\Omega$ is a connected subgroup of $G$ and $a^{b}$ is given by $b a b^{-1}$ (See [3] or [5]). Now we assume that $(\Omega, G)$ is a representation of type $B$. Then the map from $\Omega$ to $a^{\Omega}$ given by $x \rightarrow a^{x}$ is a group homomorphism. So $a^{\Omega}$ is connected by Proposition 1.1.

Now, the set $\left\{x \in G: \forall y, z \in \Omega\left(x^{y}=x^{z}\right)\right\}$ is equal to $\left\{x \in G: \forall y \in \Omega\left(x^{y}=x\right)\right\}$ if $(\Omega, G)$ is of type $A$ and is equal to $\left\{x \in G: \forall y \in \Omega\left(x^{y}=e\right)\right\}$ if $(\Omega, G)$ is of type $B$. Note that $x^{e}=e$ in the latter case. It is easy to check in each case that it is $\Omega$-subgroup and if it coincides with $G$ then the representation is a trivial one. Now we get the theorem by Lemma 2.2.

As the algebras over an infinite field of characteristic zero is connected as additive groups, we get the following:

COROLlARY 1. For finite dimensional representations of algebras over algebraically closed fields of characteristic zero, the simplicity is preserved by ele- 
mentary equivalence.

COROLlaRy 2. For a ring $R$ of finite Morley rank such that $R \cdot R \neq(0)$, if it is definably simple then it is simple.

PROOF. If we are dealing with right or left ideals (this is sufficient for many cases) then we get the theorem immediately from Theorem A and Lemma 2.1. In the case we have to deal with both-sided ideals, use Lemmas 2.1 and Lemma 2.2 again. Note that for each $x \in R, x R$ and $R x$ are connected and both contain 0 , and thus $x R \cup R x$ is indecomposable.

We cannot drop the assumption $R \cdot R \neq(0)$ in Corollary 2, because the ring $(\boldsymbol{C},+, *)$ defined by $x * y=0$ for each $x, y \in \boldsymbol{C}$ where $\boldsymbol{C}$ is the set of complex numbers is definably simple but not simple. Note also that if a ring $R$ such that $R \cdot R=(0)$ is simple then it must be finite since every subgroup of $(R,+)$ is an ideal of $R$. Hence we get:

COROLLARY 3. For rings of finite Morley rank, the simplicity is preserved by e'ementary equivalence.

We thank the referee to point out that we do not need the rings to be infinite in Corollary 3 and to give a correct example after Corollary 2.

All the results we gave so far can be proved in the superstable case instead of finite Morley rank case by slightly modifying them if necessary using methods in [3]. A proof for the preservation of simplicity of a ring by elementary equivalence in the superstable case will be given somewhere.

Now, we give our final theorem in this paper. Proof goes almost parallel to that for groups $([9])$.

Theorem B. Any (definably) simple ring of finite Morley rank is almost strongly minimal. In particular, it is $\omega_{1}$-categorical.

Proof. Let $D$ be a strongly minimal formula. Let $H_{0}$ be the minimal subgroup such that $D \cap C$ is infinite for some coset $C$ of $D$. It is easy to verify that $D \cap C$ is indecomposable. Choose some element $a$ of $D \cap C$ and put $S_{0}=(-a)+(D \cap C)$. By Lemma 2.1, the ideal generated by $S_{0}$ is definable and algebraic over $S_{0}$ and a finite number of constants. Hence it is algebraic over $D$ and some finite number of constants and, on the other hand, coincides with the whole ring. 
COROLlary 4. Any finite dimensional simple algebra over an algebraically closed field is almost strongly minimal.

Note that for an algebra over a field, simplicity is same whether if it is considered as an algebra or a ring.

There is another first order formulation of groups with an operator domain. If $\Omega$ is an operator domain of a group, $L_{\Omega}$ will denote the language obtained by adding each element of $\Omega$ as a unary function symbol to the language of groups $L$. If $G$ is a simple $\Omega$-group then it must be the prime model in the language $L_{\Omega}$. So we cannot talk about the preservation of simplicity and we do not know even whether or not being definably simple is preserved by elementary extensions. But we can prove the almost strong minimality if it is (definably) simple and has some strongly minimal formula over it. This can be satisfied if it is weakly categorical ([9]) for example. So, as a corollary, any finite dimensional representation of a group or a ring $\Omega$ is almost strongly minimal with respect to the language $L_{\Omega}$. In particular, it is $|\Omega|^{+}$-categorical.

\section{References}

[1] Baldwin, J. T., Almost strongly minimal theories. I, J. Symbolic Logic, 37 (1972), 487-493.

[2] Baldwin, J. T. and Lachlan, A.H., On strongly minimal sets, J. Symbolic Logic, 36 (1971), 79-96.

[3] Berlin, Ch. and Lascar, D., Superstable groups, Ann. Pure Appl. Logic, 30 (1986), $1-43$.

[4] Humphreys, J.E., Introduction to Lie Algebras and Representation Theory, Springer, New York, 1972.

[5] Nesin, A., Zil'ber's results, preprint.

[6] Rose, B.I., On the model theory of finite-dimensional algebras, Proc. London Math. Soc., 40 (1980), 21-39.

[7] Shelah, S., Classification Theory and the Number of Non-Isomorphic Models, NorthHolland, Amsterdam, 1978.

[8] Zil'ber, B.I., Rings with $\omega_{1}$-categorical theories, Algebra and Logic, 13 (1974), 95-104.

[9] Zil ber, B.I., Groups and rings with categorical theories, Fund. Math., 95 (1977), 173-188 (Russian).

Hirotaka KIKYo

Department of Mathematics

School of Science and Engineering

Waseda University

Okubo, Shinjuku-ku, Tokyo 160 